It involves the existence of a complete differential of the functions $u$ and $v$. Cf. Stolz, Differential und Integralrechnung, vol. 1 , ch. $4, \S 8$ and vol. 2 , ch. $12, \S 7$.

To test the truth of a general theorem one mode of procedure is to form an example which proves the theorem to be false. From the foregoing it appears that in the present case simpler examples are surely impossible than those

(a) in which $f^{\prime}(z)$ becomes discontinuous along a curve whose tangent does not turn continuously along any arc ; $*$

(b) in which, $f^{\prime}(z)$ being assumed to remain finite in $T$, $f^{\prime}(z)$ becomes discontinuous in a Cantor's set of positive content ;

(c) in which not both of the partial derivatives $\frac{\partial u}{\partial x}, \frac{\partial u}{\partial y}$ are capable of surface integration over any region lying in $T$, or of line integration along a line parallel to one of the coördinate axes.

HARVARD UNIVERSITY, September, 1898.

\title{
THE FIFTIETH ANNIVERSARY MEETING OF THE AMERICAN ASSOCIATION FOR THE ADVANCEMENT OF SCIENCE.
}

The semi-centennial meeting of the American Association for the Advancement of Science was held in Boston, August 22-27. There were over 900 members and associates registered. A large number of members of affiliated societies were also in attendance, many of whom did not register, but had the privilege of taking part in the proceedings of the sections in which they were interested. Their presence increased the general scientific interest; and it was the evident desire of the various sections to have still closer relations with the respective affiliated societies, as it is felt that any tendency towards the isolation of groups of specialists may partially defeat one of the objects the Association has at heart, viz., the spread of a popular interest in the work of scientific men.

The section of mathematics and astronomy, and its near neighbor, the physical section, were well attended, the

* It is possible that by analysis similar to Jordan's, Cours d'Analyse, vol. $1, \& \& 193-196$, this condition may be made more general. 
programmes being fairly representative of the various phases of American thought and activity in the mathematical and physical sciences.

The officers of Section A were: Vice-President, E. E. Barnard ; Secretary, Winslow Upton; Councillor, E. C. Pickering; Sectional committee, E. E. Barnard, W. Upton, J. McMahon, C. L. Doolittle, H. M. Paul, G. E. Hale; Committee to nominate officers of section, E. E. Barnard, W. Upton, G. C. Comstock, J. R. Eastman, R. S. Woodward.

The opening address of the Vice-President was on "The development of astronomical photography."

Of the various contributions accepted by the committee twelve are in pure mathematics, thirteen in applied mathematics, and sixteen in astronomy. A few of the titles that appeared on the preliminary programme are not reproduced here on account of the absence both of the authors and of the finished papers. For convenience of grouping, the numbers are rearranged as follows :

(1) "Graphical logic." By Professor Ellen Hayes, Wellesley College, Wellesley, Mass.

(2) "On the operation groups of order 48 and those of order $2 p^{3}, p$ being any prime number." By Dr. G. A. MrLLER, Cornell University, Ithaca, N. Y.

(3) "Illustrations of the comitant method of constructing the imaginary loci of analytical geometry, so as to render their properties evident to the eye." By Professor FraNK H. Loun, Colorado College, Colòrado Springs.

(4) "On the aims of the international society for the promotion of quaternions and allied branches." By Dr. Alexander Macfarlane, Lehigh University, South Bethlehem, Pa.

(5) "Linear transformations in four dimensions." By Professor Arthur S. Hathaway, Rose Polytechnic Institute, Terre Haute, Ind.

(6) "On rational right-angled triangles, II." By Dr. Artemas Martin, U. S. Coast Survey, Washington, D. C.

(7) "Some nutes on 'direction.", By S. Edwakd WarREN, C.E., Newton, Mass.

(8) "The general theory of anharmonics." By Professor Edgar Odell Lovetw, Princeton University, Princeton, N. J.

(9) "A ternary and a quaternary linear group simply isomorphic to the group of linear fractional substitutions in the Galois field of order $p^{n}$." By Dr. Leonard Eugene Dickson, University of California, Berkeley, Cal. 
(10) "On harmonic functions." By Dr. Rollin A. Harris, U. S. Coast and Geodetic Survey.

\section{HARRIS.}

(11) "A proposed tidal analyzer." By Dr. Rollin A.

(12) "A tidal abacus." By Dr. Rollin A. Harris.

(13) "The harmonic analysis of high and low waters." By Dr. Rollin A. Harris.

(14) "The mass and moments of inertia of the earth's atmosphere." By Professor R. S. Woodward, Columbia University, New York City.

(15) "Two new forms of apparatus for measuring the acceleration of gravity." By Professor R. S. WoodwARD.

(16) "The gravitation constant and the mean density of the earth." By Professor R. S. WoodwaRd.

(17) "The limitations of the present solution of the tidal problem." By JoHN F. HAyford, Coast and Geodetic Survey, Washington, D. C.

(18) "Fifty years of American geodesy." By E. D. Preston, Coast and Geodetic Survey, Washington, D. C.

(19) "On the duplex base apparatus of the U. S. Coast and Geodetic Survey." By William Ermbeck, U. S. Coast and Geodetic Survey, Washington, D. C.

(20) " On the diminution of the refraction of the atmosphere with height and its effect upon trigonometrically determined elevations from reciprocal zenith distances." By William Eimbeck.

(21) "Behavior of the atmospheres of gas- and vaporgenerating globes in celestial space." By Dr. J. WoodBridge Davis, Woodbridge School, New York City.

(22) "Making astronomy popular." By Miss MARY Proctor, New York City.

(23) "Correction of local error in stellar photometry." By Henry M. Parkhurst, Brooklyn, N. Y.

(24) "Variation of latitude at New York City and the constant of aberration from observations with a zenith telescope (Talcott's method) at Columbia University Observatory during the years of 1892-1898." By Professors J. K. REes, Harold Jacoby, and Dr. H. S. Davis.

(25) "The parallaxes of $61^{1}$ and $61^{2}$ Cygni from a reduction of the Rutherfurd measures." By Dr. H. S. Davis, Columbia University Observatory.

(26) "The Præsepe group : measurementand reduction of the Rutherfurd photographs." By Dr. Frank SchlesINGER, Columbia University:

(27) "Discordances between the north polar distances of stars derived from direct and reflected observations." By 
Professor J. R. Eastman, U. S. Naval Observatory, Washington, D. C.

(28) "The treatment of results from reflection observations at the Greenwich observatory." By Professor J. R. Eastman.

(29) "A description of the altazimuth instrument recently constructed for the U. S. Naval Observatory." By George A. Hill, Washington, D. C.

(30) "On a new application of the prismatic camera to total eclipse." By Professor David P. Todd, Amherst College, Amherst, Mass.

(31) "A description of instantaneous azimuth and altitude charts of the heavens." By F. W. CoAr, Cambridge, Mass.

(32) "Instruction in elementary astronomy by means of observations made by the student." By W. MAxwell REED, assistant in astronomy, Harvard College.

(33) "Personal equations during the past century; a brief summary." By Professor Truman H. Safford, Williams College, Williamstown, Mass.

(34) "The condition of the surface of the planet Jupiter." By Professor G. W. HougH, Dearborn Observatory. (With lantern slides.)

(35) "The Yerkes Observatory and its work." By Professor Geo. E. Hale, Director of the Yerkes Observatory, Williams Bay, Wis. (With lantern slides.)

(36) "A summary of planetary work at the Lowell Observatory and the conditions under which such work has been performed." By A. E. Douglass, Lowell Observatory, Flagstaff, A. T.

(37) "Astronomy in southern California." By Dr. Lewrs

SwIFT, Lowe Observatory, and Echo Mountain, Cal.

(38) "Report on the recent progress in hydrodynamics." By Professor Ernest W. Brown, Haverford College, Haverford, $\mathrm{Pa}$.

(39) "Report on theory of invariants: the chief contributions of a decade." By Professor Henry S. White, Northwestern University, Evanston, Ill.

(40) "Report on the recent progress in the mathematical theory of electricity and magnetism." By Professor Arthur G. Webster, Clark University, Worcester, Mass.

(41) "Report on the modern group theory:" By Dr. G. A. Mrller, Cornell University, Ithaca, N. Y.

In No. 1 it is pointed out that Mill's fifth "canon of induction" is essentially a mathematical proposition and is 
easily presented by means of the Cartesian graph. The ordinary axes of reference must be viewed as cause-effect axes which become specialized for any particular subject of investigation. In the upper right-hand quarter of the field surrounding the origin is a region which may be called the field of observation. It represents the investigator's limits of experiment. Exterior to the observation field is the field of inference. The curve expressing the concomitant variations of two phenomena-whatever kind of curve it may bebecomes inferential after crossing the boundaries of the field of observation. There is a generic cause-effect curve possessing properties which belong to specific curves, but these properties cannot be incorporated into the abstract equation of pure mathematics $f(x, y)=0$. The full paper will appear in Science.

In No. 2 it is shown that there are only two groups of order 48 that do not contain any subgroup of order 24. Each of these two groups includes 16 conjugate subgroups of order 3 and only one subgroup of order 16. This subgroup is characteristic as well as Abelian. In one of the two groups it contains 15 operators of order 2 and in the other it contains 12 operators of order 4 that do not have a common square. The total number of the groups of order 48 is 52 . Since $48=3.16$ there are as many Abelian groups of this order as there are of order 16, viz., 5, and each of these groups is the direct product of an Abelian group of order 16 and some operator of order 3. There is one Hamiltonian group of order 48 . It is the direct product of the quaternion group and the cyclical group of order 16 . The second part of the paper proves that when $p$ is any odd prime number there are just 15 groups of order $2 p^{3}$. Three of them are Abelian, but none is Hamiltonian since the order of a Hamiltonian group must be divisible by 8 . Ten of the groups of order $2 p^{3}$ contain Abelian subgroups of order $p^{3}$ while only two of them contain cyclical subgroups of this order. Each group of order $2 p^{3}$. contains only one subgroup of order $p^{3}$ and five such groups contain only one subgroup of order 2. When $p=2$ there are only 14 groups of order $2 p^{3}$. These 14 groups have been known for several years. Five of them are Abelian and one is Hamiltonian. These groups now complete the list of all the groups whose orders are less than 64 .

No. 3 is a continuation of a paper read at the Madison meeting, 1893, and in the interim an article has appeared 
in the Annals of Mathematics, vol. 8, No. 2, under the title "A construction for the imaginary points and branches of plane curves," wherein the earlier devices for the representation of imaginary loci are discussed, and the theoretical relationships of the author's method pointed out. The present paper deals with its pedagogical usefulness. Suppose that a beginner in analytic geometry learns first the usual (Argand's) construction for complex quantities, their addition, multiplication, etc., and is then taught to define the point $x, y$ as located by the complex number $x+i y$. This coincides with the Cartesian definition if the coördinates $x$ and $y$ are real, and equally permits the construction of the point if they are imaginary or complex. A linear equation $a x+b y+c=0$ is satisfied by one and only one pair of coordinates locating any given point of the plane. Its locus accordingly covers the plane with a single continuous sheet. If $p+i q$ represent the value of $x$ at any point, those points for which $q=0$ all lie in a right line, which is the familiar real locus provided $a, b, c$ be real. All points for which $q=1$ lie on a parallel to this, those for which $q=2$ on a second, etc. These parallels are called "comitants,"-viz : they are the " $x$-comitant 1 ," the " $x$-comitant 2 ," etc. There is a similar series of $y$-comitants. If $a, b, c$ are complex, the one series of equidistant parallels is crossed by the other, the two "comitants zero" meeting at the real point. The locus of an equation of second degree is in general two leaved, exemplifying the elementary properties of Riemann's surfaces. The foci are branch points. The comitants are curves of second, third, or fourth order, but points on them are very simply located by direct use of the equation. Diagrams were exhibited illustrating comitants of real and imaginary right lines, and of the three species of conic, with cases of intersection and contact at imaginary points.

In No. 4 Dr. Macfarlane said in part that the province of mathematics which it is proposed to develop and promote is more or less fully covered by such names as quaternions, Ausdehnungslehre, vector-analysis, geometric calculus, directional calculus, space-analysis, etc. These various titles have been used by writers on the subject; what is common to them all is that the branch of analysis treated of is founded on the properties of space. According to the view of the speaker, ordinary algebra is the analysis of the straight line, or anything that can be adequately represented by the straight line; the algebra of the complex quantity is the analysis of the plane, or anything that can be adequately 
represented in a plane, that is, analytical plane trigonometry; space analysis is the algebra of quantities in space, or anything that can be adequately represented in space. The elementary part of space analysis consists of analytical spherical trigonometry and is identical with Hamilton's quaternions. The logical connection of quaternions with analytical plane trigonometry was pointed out, the argument being, that, if the latter is algebra, so also is the former. A complementary part of the analysis, undeveloped by Hamilton, deals with analytical hyperboloidal trigonometry. The speaker said that while analytical plane trigonometry corresponds to Euclidean geometry, the spherical and hyperboloidal trigonometries correspond to the two non-Euclidean geometries. From these and other considerations the author contended that the branch of mathematics which it is proposed to develop is not liable to the objection of " being within very narrow bounds," an objection which was given out at a meeting of the German Mathematical Society. On the contrary, space analysis, being the logical generalization of the algebra of the complex quantity, must be of the widest influence in the whole realm of mathematical analysis. 'The greatest obstacle to progress at present existing is needless diversity of notation ; to promote arrangements for arriving at a settled notation is one of the first objects of the society named in the title.

No. 5 applies quaternion analysis to the treatment of continuous transformations that are equivalent to rigid, dilational, and inversional transformations of a fourfold figure. These are shown to be the most general transformations in which corresponding figures are similar in their infinitely small parts. The normal forms, invariant figures, and path curves of these transformations are also determined. With respect to rigid motion in fourfold space it appears that motion about a point has in general no other fixed point. There are two fundamental motions about a point, called a turn and contra-turn, whose invariant figures are a system of planes through the center, so that one and only one passes through a given central line. The path curves are circles in these planes and all lines are turned through the same angle. Every rigid motion about a fixed point is a combination of a turn and contra-turn. It has two invariant planes, in which every line of one plane is perpendicular to every line of the other. The angle between corresponding lines in one of these planes is the sum, and in the other the difference, of the angles of the 
component terms. The path curves of points in these planes are circles, and of points outside these planes spiral curves on circular rings in the boundaries of fourfold spheres that are concentric with the fixed point. When two points are fixed, the angles of the component turns are equal, every point of one invariant plane becomes fixed, and the motion is rotation about this plane as axis. The parallels to the other plane become invariant equators. The path curve of a point is a circle that is concentric with the axis and lies in an equator. The rigid displacement with no point fixed is the resultant of a rotation about a fixed axis and a translation parallel to that axis. It is interesting to note that, as in a plane, symmetric figures as to a center are rigid displacements of each other. Thus a person could be moved rigidly out into fourfold space and back, so as to appear on his return as his image in a mirror appears.

No. 6 proves several properties of right triangles stated in part I of the paper, read last year. It also proves the following two theorems: Every odd number greater than 1 is the shorter leg of a right triangle whose sides are integers prime to each other. The double of any even number except 2 is the shorter leg of a right triangle whose sides are integers prime to each other. The following five problems are fully solved: "To find all the right triangles whose sides are integers, having one leg equal to 60.' 'Thirteen such triangles.) "To find any number of rational right triangles having a common leg." [The general expressions for the sides of two such triangles are

$$
\begin{aligned}
& 2 p q r s, \quad p q\left(r^{2}-s^{2}\right), \quad p q\left(r^{2}+s^{2}\right) ; \\
& \left.2 p q r s, \quad r s\left(p^{2}-q^{2}\right), \quad r s\left(p^{2}+q^{2}\right) .\right]
\end{aligned}
$$

"To find any number of right triangles having the same hypotenuse." "To find a right triangle whose sides and area are integers in arithmetical progression." "To find right triangles whose legs are consecutive integers." [If $a, b, c$ be one solution, the next solution in order of magnitude is $2(a+c)+b, 2(b+c)+a, 2(a+b)+3 c]$. The paper will be printed in the Mathematical Magazine.

No. 7 argues chiefly against the proposed disuse of the term "direction." The idea of single direction is gained by standing at one point and intently facing another. It is which way one point is from another. Turning from facing 
one point to facing another we get the idea of difference of direction, or an angle. When by natural movements of work or play, we become familiar with the terms forward and backward, right and left, up and down-each pair representing the pair of opposite senses in which a single line of direction can be taken-we have, in the concrete terms of daily life, the standard guiding directions with which all others are compared. These three constitute a system such that no fourth direction has the same relation to them that they have to each other, viz., the relation that motion on any one of them involves no progress in the direction of the other two. The difference between these directions is the right angle, and each is perpendicular to the others. Dimensions are measures in the independent directions. Moving invariably from one point in the direction of another fixed point, my path has but one direction and is a straight line. Sometimes the word "direction" is avoided and a straight line is defined as the shortest distance between two points; but the best definition should make use of the primary property and not a property which, however elementary, is a consequence of the primary one. Thus, let $C$ be a point not on the line $A B$, and $C D$ the perpendicular from $C$ to $A B$. We may go from $A$ to $B$ in the direction $A B$, or we may go from $A$ to $B$ through $C$. In the latter case we make the same amount of progress as before in the direction $A B$, and make besides a progress in the independent direction $D C$, and back again : hence the path in the single direction $A B$ is clearly seen to be shorter. Another definition is given by a recent ingenious writer, who says, "A straight line is a line which pierces space evenly, so that a piece of space from along one side of it will fit any side of any other portion." But what can the word "evenly," used with definite precision, mean if not "in one direction"? And if used with precision what is the use of the explanatory clause following it? The paper also alludes to broken lines, curves, parallels, etc., and concludes that there is no sufficient reason for rejecting the old familiar term "direction" which tells "which way," and with precision in the denominate numbers of angular measure, as "distance" tells "how far," and with precision in the denominate numbers of linear measure.

No. 8 will probably be printed in full in the Bulletin. A summary is not at present available.

No. 9 is connected with a paper, recently presented to the 
London Mathematical Society, which determines the order, generators and structure of the linear homogeneous groups in the Galois field of order $p^{n}$ that have the quadratic invariants

$$
\sum_{i=1}^{m} \xi_{i} \eta_{i}, \quad \xi_{1}+\eta_{1}+\sum_{i=1}^{m} \xi_{i} \eta_{i}
$$

For the special case $p^{n}=2$, these groups are the first and second hypoabelian groups of Jordan. The present paper discusses the quaternary group $G$ leaving invariant

$$
\xi_{1}+\eta_{1}+\xi_{1} \eta_{1}+\xi_{2} \eta_{2}
$$

For $p>2$, every substitution of $G$ of determinant +1 can be given the form

$$
\left(\begin{array}{rrrr}
\nu \alpha \delta & -\nu \beta \gamma & \nu \alpha \beta & \nu \gamma \delta \\
-\nu \beta \gamma & \nu \alpha \delta & -\nu \alpha \beta & -\nu \gamma \delta \\
\nu \alpha \gamma & -\nu \alpha \gamma & \nu \alpha^{2} & \nu \gamma^{2} \\
\nu \beta \delta & -\nu \beta \delta & \nu \beta^{2} & \nu \delta^{2}
\end{array}\right) \equiv\left[\begin{array}{c}
\alpha \gamma \\
\beta \delta
\end{array}\right]_{\nu},
$$

where $\nu(\alpha \delta-\beta \gamma)=1$. Two such substitutions compound as follows :

$$
\left[\begin{array}{ll}
\alpha^{\prime} & \gamma^{\prime} \\
\beta^{\prime} & \delta^{\prime}
\end{array}\right]_{\nu^{\prime}} \cdot\left[\begin{array}{ll}
\alpha & \gamma \\
\beta & \delta
\end{array}\right]_{\nu}=\left[\begin{array}{ll}
\alpha^{\prime} \alpha+\beta^{\prime} \gamma & \gamma^{\prime} \alpha+\delta^{\prime} \gamma \\
\alpha^{\prime} \beta+\beta^{\prime} \delta & \gamma^{\prime} \beta+\delta^{\prime} \delta
\end{array}\right]_{\nu^{\prime} \nu}
$$

Hence those substitutions in which $\nu$ is a square (which may be supposed to be unity) form a quaternary linear group simply isomorphic to the simple group of linear fractional substitutions of determinant $\alpha \delta-\beta \gamma=1$. Hence $G$ has the factors of composition $2,2, \frac{1}{2} p^{n}\left(p^{2 n}-1\right)$. A like result holds for the ternary orthogonal group [see the BuLceTrN for May, 1898, p. 388]. For $p=2, n>1$, the group $G$ has a subgroup of index two simply isomorphic to the linear fractional group in the $G F\left[2^{n}\right]$, so that $G$ has the factors of composition $2,2^{n}\left(2^{2 n}-1\right)$.

No. 10 points out some connections which exist between various particular solutions of Laplace's equation, and shows how these may, in a certain sense, be regarded as a natural outgrowth of the harmonic functions with which the modern theory of functions has to deal. A few analogous equations are embraced in the two forms considered, the latter being 


$$
\begin{gathered}
\begin{array}{c}
\partial^{2} V \\
\partial x^{2}
\end{array}+\frac{\partial^{2} V}{\partial y^{2}}+\frac{\partial^{2} V}{\partial z^{2}}+\cdots=0 \\
\frac{\partial^{2} U}{\partial x^{2}}-\left(\begin{array}{l}
\partial^{2} U \\
\partial y^{2}
\end{array}+\frac{\partial^{2} U}{\partial z^{2}}+\cdots\right)=0 .
\end{gathered}
$$

A number of theses or statements, self-evident or nearly so, are laid down at the outset; and the paper consists largely in their application. One thesis, for instance, is : Given any solution of (1) or (2) of the form

$f_{0}(y, z, \cdots) \chi(x)+f_{2}(y, z, \cdots) \chi^{\prime \prime}(x)+f_{4}(y, z, \cdots) \chi(x)+\cdots$, and such that the entire coefficients of $\chi^{\prime \prime}(x), \chi^{\text {iv }}(x), \cdots$ in (1) or (2) are severally zero ; then is

$f_{0}(y, z, \cdots) \psi(x)+f_{2}(y, z, \cdots) \psi^{\prime \prime}(x)+f_{4}(y, z, \cdots) \psi^{\text {iv }}(x)+\cdots$ a solution, $\psi$ being an arbitrary function. $E . g ., 1 / r$ which is a solution of (1) may be thus expanded by the binomial theorem :

$\frac{1}{r}=\frac{1}{x}-\frac{1}{2} \frac{y^{2}+z^{2}}{x^{3}}+\frac{1 \cdot 3}{2 \cdot 4} \frac{\left(y^{2}+z^{2}\right)^{2}}{x^{5}}-\frac{1 \cdot 3 \cdot 5}{2 \cdot 4 \cdot 6} \frac{\left(y^{2}+z^{2}\right)^{8}}{x^{7}}+\cdots$. If $\chi(x) \equiv \frac{1}{x}$, this may be written

$\frac{1}{r}=\chi(x)-\frac{1}{2} \frac{1}{2 !}\left(y^{2}+z^{2}\right) \chi^{\prime \prime}(x)+\frac{1 \cdot 3}{2 \cdot 4} \frac{1}{4 !}\left(y^{2}+z^{2}\right)^{2} \chi^{\mathrm{iv}}(x)-\cdots$.

Now let $\psi(x) \equiv \sinh x$, and we have

$$
\sinh x\left[1-\frac{1}{2^{2}}\left(y^{2}+z^{2}\right)+\frac{1}{2^{2} 4^{2}}\left(y^{2}+z^{2}\right)^{2}-\cdots\right]
$$

or $\sinh x J_{0}(\bar{\omega})$ as another solution of Laplace's equation. Again, if $\psi(x) \equiv x^{n}$, we have

$$
\begin{gathered}
x^{n}\left[1-\frac{n(n-1)}{2^{2}} \frac{y^{2}+z^{2}}{x^{2}}\right. \\
\left.+\frac{n(n-1)(n-2)(n-3)}{2^{2} 4^{2}} \frac{\left.\left(y^{2}+z^{2}\right)^{2}-\cdots\right],}{x^{4}}-\cdots\right]
\end{gathered}
$$

or, putting $x=r \mu$,

$$
\begin{gathered}
r^{n} \mu^{n}\left[1-\frac{n(n-1)}{2^{2}} \frac{1-\mu^{2}}{\mu^{2}}\right. \\
\left.+\frac{n(n-1)(n-2)(n-3)}{2^{2} 4^{2}} \frac{\left(1-\mu^{2}\right)^{2}}{\mu^{4}}-\cdots\right]
\end{gathered}
$$


which is $r^{n} P_{0}(\mu)$, as another solution. In a similar manner, the solution $(y+\sqrt{-1 z})^{n} / r^{2 n+1}$ gives, upon making

$$
\begin{gathered}
\chi(x)=1 / x^{2 n+1} \text { and } \psi(x)=\sinh x, \\
\sinh x(\cos n \varphi+\sqrt{-1} \sin n \varphi) J_{n}(\bar{\omega})
\end{gathered}
$$

as a solution of (1). When concerned with (2), we may start with the solution $1 / \sqrt{x^{2}-\left(y^{2}+z^{2}\right)}$, and proceed as before but making $\psi(x)$ equal to $\sin x$ or $\cos x$ instead of $\sinh x$. If $\varphi(\alpha x+\beta y+\gamma z+\cdots)$ be expanded by Taylor's theorem in powers of $\beta y+\gamma z+\cdots,(1)$ will be satisfied if $\alpha^{2}+\beta^{2}+\gamma^{2}+\cdots$ $=0$, and $(2)$, if $\alpha^{2}-\left(\beta^{2}+\gamma^{2}+\delta^{2}\right)=0$. Such expansion satisfies the requirements of the above-quoted thesis. By varying $\varphi, \alpha, \beta, \gamma, \cdots$ a great number of particular solutions will be obtained.

$\varphi(\alpha x+\beta y)$ where $\alpha^{2}+\beta^{2}=0$, or $\varphi(\alpha x+i b y)$ where $a^{2}=b^{2}$ and $i^{2}=-1$, is a solution of $(1)$; this solution breaks up into two, the 1-terms and the $i$-terms. Similarly $\varphi(\alpha x+\beta y)$ where $\alpha^{2}-\beta^{2}=0$, or $\varphi(a x+\mathrm{i} b y)$ where $\alpha^{2}=b^{2}$ and $\mathrm{i}^{2}=+1$, is a solution of (2); this solution breaks up into two, the 1 -terms and the i-terms. So $\varphi(\alpha x+\beta y+\gamma z)$ where $\alpha^{2}+\beta^{2}+$ $\gamma^{2}=0$, or $\varphi(a x+i b y+j c z)$ where $a^{2}=b^{2}+c^{2}$ and $i^{2}=j^{2}=$ -1 , is a solution of (1); the $1-, i-, j-, i j$-parts of this solution are likewise solutions. Again, $\varphi(\alpha x+\beta y+\gamma z)$ where $\alpha^{2}-\left(\beta^{2}+\gamma^{2}\right)=0$, or $\varphi(a x+\mathrm{i} b y+\mathrm{j} c z)$ where $a^{2}=b^{2}+c^{2}$ and $i^{2}=j^{2}=+1$, is a solution of $(2)$ which can be broken up into four solutions. The expansion $\varphi(\alpha x+\beta y+\gamma z+\delta \omega)$ breaks up into eight solutions of (1) if $\alpha^{2}+\beta^{2}+\gamma^{2}+\delta^{2}=0$, and of (2) if $\alpha^{2}-\left(\beta^{2}+\gamma^{2}+\delta^{2}\right)=0$; in the first case they are the 1-, $i-, j-, k-, i j-, i k-, j k-, i j k$-parts, where $i^{2}=j^{2}=k^{2}=$ -1 ; and in the second, like parts where $i^{2}=j^{2}=k^{2}=+1$. As an illustration, suppose $\varphi=$ cosine, $a^{2}=1, b^{2}=\frac{1}{2}, c^{2}=\frac{1}{2}$; then the 1-terms in $\varphi(a x+i b y+j c z)$ or

$$
\cos \left(x+\frac{i}{\sqrt{2}} y+\frac{j}{\sqrt{2}} z\right)
$$

give $\cos x \cosh \frac{y}{\sqrt{2}} \cosh \frac{z}{\sqrt{2}}$ as a solution of Laplace's equation. This when multiplied by the time factor denotes the velocity-potential of a standing wave in a V-shaped canal whose sides make angles of $45^{\circ}$ with the vertical.

The object of No. 11 is to show the feasibility of constructing a machine such that if into it the hourly heights 
of the sea be entered once, they will be distributed and added in the various ways required in tidal work. Nearly all of the many wheels used are of two kinds or patterns, a fact which would be of great importance should the construction of such a machine ever be attempted. A brief description of this analyzer will appear in Appendix No. 9, of the U. S. Coast and Geodetic Survey Report for 1897.

"Tidal Abacus" is a term applied in No. 12 to an apparatus consisting of a set of pairs of curves drawn upon movable boards together with a rack upon which they are mounted. The object of this contrivance is to enable a person to ascertain, by mechanical or graphic means, the height of the tide, or surface of the sea, at any given time past or future. The curves represent the harmonic components of the tide at the station for which they are drawn, and so the result of properly superposing them should agree with that obtained from computation or from a tide-predicting machine. A description of this apparatus is given in the Survey Report for 1894 (p. 183) ; but it was exhibited, for the first time, before the recent meeting at Boston. The tide represented by the curves is that at Port Townsend, Washington, where the amplitude of the diurnal wave often exceeds that of the semidiurnal.

The process outlined in No. 13 consists essentially in supplying by interpolation (without drawing the tide curve) the hourly heights of the semidiurnal portion of the curve. These heights are summed and analyzed for the semidiurnal components as if they were true heights of the surface of the sea. Knowing from observations the times and heights of the actual high and low waters and having all hourly heights of the semidiurnal tide supplied as just stated, the former heights when diminished by the latter give points on the diurnal curve. These are summed and analyzed for the diurnal components as if they were true heights of the surface of the sea. This method will be described in Appendix No. 9 of the Survey Report for 1897.

No. 14 showed how to determine upper and lower limits to the mass and moments of inertia of the earth's atmosphere. Expressing results in round numbers the investigation shows that the mass of the atmosphere must be greater than $1 / 1000000$ th and less than $1 / 1200$ th of the entire mass of the earth. Similarly the moments of inertia of the atmosphere must be greater than $1 / 555000$ th and less than 
$1 / 200$ th of the corresponding moments of the entire earth. This paper will be published in full in the Astronomical Journal.

No. 15 described two new forms of apparatus for measuring the acceleration of gravity. The first of these consists of a helical spring held rigidly at its upper end and vibrating vertically under the action of a mass attached at the lower end. Calling the mass of the spring $m$, the attached mass $\mu$, the static elongation of the spring (under $\mu$ ) $\varepsilon$, the periodic time of vibration $\tau$, the acceleration of gravity is found to be

$$
g=\varepsilon \frac{\mu+\frac{1}{3} m}{\mu+\frac{1}{2} m}\left(\frac{2 \pi}{\tau}\right)^{2}
$$

This formula ignores the effect of damping, of which, however, account may be easily taken if essential. It was pointed out that this apparatus and formula afford a useful class-room and laboratory method of measuring $g$. Usually, for such work, the ratio $\mathrm{m} / \mu$ may be neglected, thus giving an approximation to $g$ independently of $\mu$. The other form of apparatus consists of a long piece of steel tape suspended vertically, being fastened rigidly at its upper end and carrying a relatively large mass at its lower end. The elastic pendulum thus formed will vibrate laterally and longitudinally. It can be shown that the effect of the latter vibration will be to produce a small modification of the lateral vibration for which correction can be applied, so that the value of $g$ is given by a formula similar to that for a rigid pendulum. It was explained that both forms of apparatus may be enclosed in receivers from which the air may be exhausted and in which the temperature may be fixed by means of melting ice.

No. 16 called attention to the need of further researches to determine the gravitation constant and the mean density of the earth. It was shown that their product is not known with considerable precision and that we may hope to find their quotient. The importance of additional measurements of the gravitation constant was especially urged.

Mr. Hayford, in summarizing his useful paper (No. 17) states that his purpose is not to present anything new ; but to point out a very weak point in tidal theory - to indicate where the most urgent tidal problem now lies. The height of the tide, referred to mean sea level, at any instant at any 
given point may be represented accurately by an expression of the form

$$
h=A_{1} \cos \left(\alpha_{1} t+\beta_{1}\right)+A_{2} \cos \left(\alpha_{2} t+\beta_{2}\right) \cdots
$$

From pure theory, reasoning from the known motions of the moon, sun, and earth, and the Newtonian law of gravitation, it has been shown that if certain definite values be assigned to the quantities $\alpha_{1}, \alpha_{2}, \cdots$ (fixing the periods of the separate terms) each term then truly represents one of the invariable components of the tide. Here, after fixing merely the periods of the separate components the contribution of tidal theory ends, and the work of direct observation at the particular station under consideration begins. Theory does not furnish even a rough approximation for any station to the values of the $A$ 's, defining the amplitudes of the separate components, nor of the $\beta^{\prime}$ s which fix the epochs. If we look at the actual tidal problem of nature it becomes evident that the reason why theory furnishes so little is that theory at present furnishes no adequate means of taking into account the effect of boundaries (bottom end shores) upon the tidal wave, although this effect is to modify the astronomical tide, computed for an ocean of uniform depth, so greatly that there is very little resemblance between the astronomical tide and the actual tide except that the periods of the separate components are identical in the two. The way in which the astronomical tide is modified by the boundaries may be indicated roughly as follows : The wave is everywhere a forced wave since the depth of the ocean is nowhere sufficient to allow the free wave to keep pace with the moon, and the great variations in depth make the lag much greater in some latitudes than in others; when one part of a wave outstrips another on account of the difference of depth a portion of the front of the wave becomes oblique and the wave acquires a northward or southward component in its motion which may ultimately cause different portions of the wave to coalesce; variations in depth produce radical changes in the amplitude of the wave-the amplitude being rapidly reduced by friction in small depths, and being greatly increased in passing from great to small depths (and decreased in the reverse case), because nearly the same amount of kinetic energy must be exhibited by a much smaller amount of water than before; one-quarter of the surface of the earth is land and the tidal wave is reflected to a grenter or less extent at every contact with the shore and the direct wave is thus modified by combination with the reflected 
wave. As a result of the extreme modifications due to the boundaries we find that the actual tidal wave, instead of progressing steadily westward around the earth once in each lunar day, fails even in the latitude in which it encounters least obstruction (just south of Australia, Africa, and South America) to make a complete circuit in even thirty-six hours; that the actual progress is to the northward rather than the westward over about one-third of the whole ocean surface (the whole Atlantic and a part of the Pacific); that the progress is to the eastward in at least two large areas (in the European Arctic ocean and near the west coast of South America); that the amplitude of the wave is continually varying during the progress of the wave between wide limits, viz., zero (nearly) and sixty feet (or more). The most important tidal problem before us at present is therefore the problem of determining the relation between the boundaries and the modifications produced by them upon the tidal wave. There is an abundance of facts at hand to be used in the investigation. This is a comparatively unworked portion of field of tidal investigation although considerable pioneer work has been done in it along purely mathematical lines. It would seem that the best chance of success lies in investigating this problem by a semi-graphic method based upon accurate charts of such a region as Chesapeake Bay in which the facts as to the tide are well determined at a large number of points and in which the direct effect of the moon and sun is small as compared with that of the wave transmitted from the ocean.

The full paper will be printed in Science.

The following is a summary of Mr. Preston's paper (No. 18): The geodetic operations in the United States executed by the Coast and Geodetic Survey fall naturally into three distinct periods. From 1807, when the work was authorized by Congress, to 1832, when it was resumed after having been delayed from various causes, may be regarded largely as an era of preparation and experiment. From 1832 to 1843 constitutes the second period, and was characterized by the beginning of work for the first time on an adequate geodetic scale. The third period comprises the period from the date of reorganization in 1843 to the present time. During the entire time between 1807 and to-day, taking away periods of inactivity occasioned by the Civil War, and other intervals when but little work was done, on account of lack of appropriations, we have fifty years of geodetic operations. During the existence of the Survey it has been three 
times under the control of the Treasury Department, twice under the Navy, and once under law requiring its personnel to be Army or Navy officers.

The results of the work to the present time are the determination of 20,500 geographical positions, besides the execution of 38,500 square miles of topography, 100,000 miles of shore line, and 93,000 miles of deep sea sounding. $\quad 4,600$ original topographic and hydrographic sheets are on file in the archives, from which considerable more than $1,000,000$ charts have been made and distributed. There are 30,000 original volumes of observations. 203 base lines have been measured, of which 19 are primary, with an average length of 9,892 meters, and a probable error of 1/445,000 part. The three kinds of base-measuring apparatus have been, metallic bars, tape line, and a rod immersed in melting ice. The degrees of accuracy attained for the three years were, $1 / 1,000,000,1 / 2,000,000$, and $1 / 5,000,000$, in the order stated. The arcs already measured consist of an oblique arc of 22 degrees on the Atlantic coast, and one from the Atlantic to the Pacific on the 39th parallel of latitude. The largest triangle yet observed has sides of 133, 167 and 190 miles. The highest station occupied is over 14,000 feet.

Besides the regular astronomical work, the Coast and Geodetic Survey has devoted considerable labor to the improvement of star catalogues, made necessary by refined observations in latitude, longitude and azimuth. The average probable error of a declination from the latitude lists may be given as less than one-fourth of a second, which degree of precision enables an observer to determine his latitude from twenty pairs in one evening with an uncertainty of only \pm 10 feet. The Survey has sent out no less than 35 parties for the observation of solar eclipses, and transits of the inferior planets, and has determined the variations of latitude at three stations each one having been occupied more than a year. 5,000 miles of precise leveling have been executed, including four independent determinations of the height of St. Louis, two from the Atlantic and two from the Gulf of Mexico. Permanent magnetic observations have been in operation for five consecutive years at five distinct stations, at all of which, with one exception, self-registering apparatus has been continuously and exclusively employed. Gravity has been determined at 26 foreign stations, including points on all continents, and on many islands in the Atlantic and Pacific.

In physical hydrography work has been done in the exploration of the Gulf Stream, in the establishment of cotidal 
lines, and in determinations of ocean depths from earthquake waves, besides the regular hydrographic work of the Survey. The practical results of the surveys are shown in the publication of annual reports, the issue of charts, notices to mariners, coast pilots, tide tables, etc.

Among the projects now in hand are the re-survey of Chesapeake Bay, the measure of an arc through the United States on the 98th meridian, a line of transcontinental levels, primary triangulation from San Francisco to the Mexican boundary, and hydrographic surveys along the Atlantic seaboard, on the Pacific coast, and at the mouth of the Yukon in Alaska. An extension of the great arc of the United States into Mexico and the British possessions has been proposed by Dr. Pritchett, the present Superintendent, and diplomatic representations between the interested governments looking towards concerted action in the near future have already been made.

In the ordinary prosecution of the field work about 50 parties are employed in the course of each year. Added to this, the purely hydrographic work is carried on by a fleet of 15 vessels, of which 8 are steamers.

Tidal indicators have been erected, or are in process of erection, at New York, Philadelphia and San Francisco, which furnish the navigator at any moment at a distance of one mile with necessary information as to the character and amount of the tide.

The Coast and Geodetic Survey is also charged with the establishment of trial speed courses for ships of the Navy, and the administration of an office of Standard Weights and Measures, from which prototypes are issued to the different States.

Nos. 19-37 are fairly well described by their titles, and are not well adapted for summarizing. Many of them will appear in the Astronomical Journal or in Science. Owing to the length of the programme some of the astronomical papers had to be presented in a separate room, thus giving an opportunity for the attendance of the members of the conference of astronomers and astrophysicists, which had been previously held at Harvard Observatory.

A noteworthy feature of the programme of Section A was a series of four admirable reports on the recent progress, and the present state, of certain branches of mathematical science, prepared by special invitation of the sectional committee. Each report occupied about an hour in reading, 
and they were closely followed by large audiences at the last morning and afternoon sessions of the Section. The reports of Professors Brown and Webster were presented before a joint session of Sections $A$ and $B$, the former being read by Professor R. S. Woodward (who was at the same time invited to present his own papers $14,15,16$ to the joint session). Some of the reports will be printed in the Bulleterin and some in the Proceedings. Nos. 17, 18, 35, 36, 37 are also in the nature of reports of progress. The Council of the Association has now passed a resolution encouraging all the sectional committees to procure reports of this nature from competent scientists.

The Council elected to fellowship in the Association the following mathematicians and astronomers: Professor R. D. Bohannan, Mr. J. F. Hayford, Professor H. C. Lord, Dr. G. A. Miller, Miss Mary Proctor.

The next meeting of the Association will be held at Columbus, August, 1899, under the presidency of Professor Edward Orton. The officers elected for Section A are Dr. Alexander Macfarlane, Lehigh University, and Mr. J. F. Hayford, Coast and Geodetic Survey.

CURNELL UNIVERSTTY.

James MoMahoN.

\section{NOTES.}

The fourth regular meeting of the Chicago Section of the American Mathematical Society will beheld in room 35 of the Ryerson Physical Laboratory, University of Chicago. on Thursday and Friday, December 29th and 30th next. Titles, abstracts, and time requirements of papers to be presented should be in the hands of the secretary of the Section, Professor Thomas Holgate, Evanston, for the use of the programme committee, not later than December 10th.

At the Bristol meeting of the British Association for the Advancement of Science, Dr. Michael Foster was elected president, Professor G. H. Darwin one of the vice-presidents, and MaJor P. A. MACMAHON a member of the Council. Grants of money to the extent of about seventy five hundred dollars were appropriated to purposes of scientific research, the section of mathematics receiving one-third of the amount. The following papers were presented before 